\title{
$\beta$-Alanine Enhances Brain and Muscle Carnosine Levels in Broiler Chicks
}

\author{
Shozo Tomonaga ${ }^{1}$, Mitsuhito Matsumoto ${ }^{2}$ and Mitsuhiro Furuse ${ }^{1}$ \\ ${ }^{1}$ Laboratory of Regulation in Metabolism and Behavior, Graduate School of Bioresource and \\ Bioenvironmental Sciences, Kyushu University, Higashi-ku, Fukuoka-shi, Japan \\ ${ }^{2}$ Livestock and Grassland Research Division, NARO Kyushu Okinawa Agricultural Research Center, \\ Koshi-shi, Kumamoto-ken, Japan
}

\begin{abstract}
Carnosine and its methylated derivative anserine are dipeptides present in high levels in chicken muscles. They are antioxidants and putative neurotransmitters. If administration of $\beta$-alanine, one of the constituents of carnosine, could increase levels of these dipeptides in the brain and muscles, it may improve brain function and increase commercial values of the chicken meat. In the present study, we investigated whether orally administered $\beta$-alanine could increase these dipeptide levels in the brain, Musculus pectoralis superficialis, and plasma in broiler chicks. Broilers ( 2 days old) were given oral doses of $\beta$-alanine $(0.176,0.88,4.4$ and $22 \mathrm{mmol} / \mathrm{kg}$ ) twice a day for 5 days. Carnosine levels were dose-dependently increased in the brain, Musculus pectoralis superficialis, and plasma while no influence of anserine was detected. These results suggest that supplemental $\beta$-alanine could be effective in increasing carnosine levels in the brain and muscle of broiler chicks.
\end{abstract}

Key words: $\beta$-alanine, brain, broiler, carnosine, muscle

J. Poult. Sci., 49: 308-312, 2012

\section{Introduction}

Carnosine ( $\beta$-alanyl-L-histidine) and its derivative anserine ( $\beta$-alanyl-1-methyl-L-histidine) are high in the breast muscle (Musculus pectoralis superficialis, MPS) of chickens (Nishimura et al., 1988; Aristoy and Toldra, 2004). They are known as antioxidants (Kohen et al., 1988) and putative neurotransmitters (Tomonaga et al., 2004; Tomonaga et al., 2005a). Carnosine could enhance meat stability in storage by its antioxidant activity (Djenane et al., 2004). Not only that, carnosine seems to contribute to the nutritional quality of meat because dietary carnosine has a hypoglycemic effect (Yamano et al., 2001), hypotensive action (Niijima et al., 2002), antidepressant-like activity (Tomonaga et al., 2008), and anti-aging effects (Gallant et al., 2000). Carnosine is synthesized from $\beta$-alanine ( $\beta$-Ala) and histidine (His) by carnosine synthase (Drozak et al., 2010), and anserine is mainly formed from carnosine by a methylation reaction (Boldyrev and Severin, 1990), while Horinishi et al. (1978) reported that a purified enzyme from chickens could methylate His to generate anserine by direct synthesis. On the other hand, carnosinase, which hydrolyzed carnosine or an-

Received: December 14, 2011, Accepted: April 28, 2012

Released Online Advance Publication: June 25, 2012

Correspondence: S. Tomonaga, Laboratory of Regulation in Metabolism and Behavior, Graduate School of Bioresource and Bioenvironmental Sciences, Kyushu University, Higashi-ku, Fukuoka-shi, 812-8581, Japan. (E-mail: shozo@brs.kyushu-u.ac.jp) serine to its constituent amino acids, was confirmed to be present in rats and humans. The rate of carnosine hydrolysis is 3-4-fold higher than that of anserine (Pegova et al., 2000).

The influence of constitutive amino acids of these dipeptides, especially $\beta$-Ala, has been studied. Intraperitoneal injections of $\beta$-Ala caused increases in the carnosine concentration in the olfactory bulb in the brain and in the gastrocnemius muscle of mice. Cessation of $\beta$-Ala treatment for 5 days caused an increase of anserine concentration in the gastrocnemius muscle in mice (Margolis et al., 1985). Dietary $\beta$-Ala plus His supplementation increased carnosine concentration of type IIA and IIB muscle fibers in horses (Dunnett and Harris, 1999). In humans, supplementation of $\beta$-Ala could increase carnosine levels of the soleus and gastrocnemius muscles (Derave et al., 2007).

We have speculated that if administration of $\beta$-Ala, one of the constituents of carnosine, could increase levels of these dipeptides in the brain and muscles, it may improve brain function and increase commercial values (enhancement of storage stability and nutritional quality) of the chicken meat. To clarify this speculation, we first revealed that oral administration of $\beta$-Ala $(22 \mathrm{mmol} / \mathrm{kg}$ twice a day, from 2 days old for 5 days) increased both dipeptide concentrations in the brain but failed to increase the sum of the dipeptide concentrations in the MPS of layer-type chicks (Tomonaga et al., 2005b). Next, we revealed that dietary $\beta$-Ala $(0.5,1$, and $2 \%$ in commercial diet, from 4 weeks old for 4 weeks) enhanced the carnosine and anserine levels in the brain, but 
not muscles (MPS, Musculus pectoralis profundus and Musculus biceps femoris), in broilers (Tomonaga et al., 2006). From these results, we speculated that the methods used in these previous studies may not be appropriate for increasing the dipeptides in the chicken muscles because of the strain difference, inadequate treatment period and method for injection. In the present study, therefore, we evaluated a different method, oral administration of $\beta$-Ala $(0.176,0.88$, 4.4 and $22 \mathrm{mmol} / \mathrm{kg}$ twice a day, from 2 days old for 5 days) in broiler chicks. Among the free amino acids, in addition to the constituent amino acids, we focused on taurine. Administration of $\beta$-Ala induces tissue taurine depletion because $\beta$ Ala competes with taurine for uptake mechanisms (Hu et al., 2000; Tomonaga et al., 2005b, 2006). Furthermore, tissue taurine depletion evokes physiological dysfunctions such as growth retardation (Sturman, 1993; Hu et al., 2000). Therefore, we assumed that it is important to investigate tissue taurine depletion for the evaluation of the effect of supplemental $\beta$-Ala.

\section{Materials and Methods}

One-day-old male broiler chicks (Chanky) were purchased from a local hatchery (Kyushu Hatchery, Fukuoka, Japan). Food (Toyohashi Feed Mills Co., Ltd., Toyohashi, Japan) and water were freely accessible. The chicks were reared as a group until they were 1 day old. From 2 to 7 days old, birds were individually reared. The birds were distributed into experimental groups based on their body weight so that the average body weight of each group was as uniform as possible. Experimental procedures followed the guidance for Animal Experiments in the Faculty of Agriculture and in the Graduate Course of Kyushu University and the Law (No. 105 ) and Notification (No. 6) of the Government. $\beta$-Ala was purchased from Wako (Osaka, Japan). The birds ( 2 days old) were orally given distilled water $(10 \mathrm{ml} / \mathrm{kg}$, control) or $\beta$-Ala $(0.176,0.88,4.4$ or $22 \mathrm{mmol} / 10 \mathrm{ml} / \mathrm{kg})$ twice a day for 5 days. The number of birds used in each group was 7 or 8 . At 7 days old, all birds were killed following the anesthesia with isoflurane (Mylan Inc., Tokyo, Japan) and blood, whole brain tissue and MPS were collected. Blood taken from the jugular vein was collected into heparinized tubes. The blood was centrifuged at $10,000 \mathrm{~g}$ for $10 \mathrm{~min}$ at $4^{\circ} \mathrm{C}$ and the plasma was removed and stored at $-80^{\circ} \mathrm{C}$ until assaying.

Whole brain and MPS tissues were weighed and stored at $-80^{\circ} \mathrm{C}$ prior to amino acid analysis. For MPS weight, the right side was weighed; from this, total MPS weight was extrapolated. The method of sampling for the amino acid analysis has been described elsewhere (Tomonaga et al., 2005 ) with some modifications. The tissue (brain or MPS) was homogenized in $0.2 \mathrm{~mol} / \mathrm{L}$ ice-cold perchloric acid and the homogenate was cooled on ice for $30 \mathrm{~min}$ for deproteinization. The homogenate was centrifuged at 20,000 x g for $15 \mathrm{~min}$ at $0^{\circ} \mathrm{C}$. Next, the $\mathrm{pH}$ of the supernatant was adjusted to approximately 3.0 by adding $1 \mathrm{~mol} / \mathrm{L}$ sodium acetate. The sample was filtered through a $0.2-\mu \mathrm{m}$ filter (Millipore, Bedford, USA). The plasma was diluted with sulfosalicylic acid solution $(20 \mathrm{mg} / \mathrm{ml})$ and shaken for $15 \mathrm{~min}$. Then the homo- genate was centrifuged at $2,000 \mathrm{xg}$ for $15 \mathrm{~min}$ at $4^{\circ} \mathrm{C}$. Next, the sample was filtered through a $0.2-\mu \mathrm{m}$ filter (Millipore, Bedford, USA). Then, $40 \mu l$ of each filtrate was applied to the Amino Acid Analyzer L-8800 (Hitachi High-Technologies Co. Ltd., Hitachi, Japan). A standard L-amino acid solution was prepared by diluting a commercially available L-amino acid solution (Type AN II and Type B, Wako, Osaka, Japan) with water. Carnosine, anserine, their constituent amino acids ( $\beta$-Ala, histidine, and 1-methyl-histidine) and taurine were analyzed.

All data were statistically analyzed by regression analysis for the dose of $\beta$-Ala treatment and amino acid or dipeptide level. Statistical analysis was conducted using the commercially available StatView software package (SAS 1998).

\section{Results}

At the beginning of the experiment, the number of birds used in each group was 7 (control, 0.176, and $22 \mathrm{mmol} \beta$ Ala groups) or 8 ( 0.88 and $4.4 \mathrm{mmol} \beta$-Ala groups). However, two birds (one in the $4.4 \mathrm{mmol} \beta$-Ala group and one in the $22 \mathrm{mmol} \beta$-Ala group) died during the experimental periods. Furthermore, two samples (one in the control group and one in the $0.88 \mathrm{mmol} / \mathrm{kg} \beta$-Ala group) were lost during sampling. Therefore, for growth and various tissue parameters, the numbers of birds in each group were: control group, 7; $0.176 \mathrm{mmol} \beta$-Ala group, $7 ; 0.88 \mathrm{mmol} \beta$-Ala group, $8 ; 4$. $4 \mathrm{mmol} \beta$-Ala group, 7 ; and $22 \mathrm{mmol} \beta$-Ala group, 6 . For analysis of amino acid and dipeptide levels, the numbers of birds in each group were: control group, $6 ; 0.176 \mathrm{mmol} \beta$ Ala group, $7 ; 0.88 \mathrm{mmol} \beta$-Ala group, $7 ; 4.4 \mathrm{mmol} \beta$-Ala group, 7; and $22 \mathrm{mmol} \beta$-Ala group, 6 . In the analysis of brain carnosine, some sample levels were below the detection limit and they were not included in the analysis. Therefore, the numbers of birds in each group were: control group, 4; $0.176 \mathrm{mmol} \beta$-Ala group, $5 ; 0.88 \mathrm{mmol} \beta$-Ala group, $7 ; 4.4$ mmol $\beta$-Ala group, 7 ; and $22 \mathrm{mmol} \beta$-Ala group, 5 .

Table 1 represents the effect of $\beta$-Ala on growth and various tissue weights in broilers. There were no dose-dependent effects on all parameters.

Tables 2 and 3 indicate the effect of $\beta$-Ala on carnosine, anserine, their constituent amino acids and taurine levels in the brain, MPS and plasma of broilers. We presented only the data in which significant correlations were detected in Table 3. In the brain, MPS and plasma, there were significant positive correlations between the dose of injected $\beta$-Ala and carnosine or $\beta$-Ala levels. On the other hand, there were significant negative correlations between the dose of injected $\beta$-Ala and taurine levels in the brain, MPS and plasma.

\section{Discussion}

$\beta$-Ala dose-dependently increased carnosine levels in the brain and MPS of broiler-type chicks. These results were consistent with our previous study using the same treatment in layer-type chicks (Tomonaga et al., 2005b). On the other hand, the effect was somehow different in anserine metabolism: brain and MPS anserine levels were not influenced (brain level was increased and MPS level was decreased in 
Table 1. Effect of $\beta$-alanine on growth and various tissue weights in broiler chicks

\begin{tabular}{lcrrrr}
\hline \hline & \multicolumn{5}{c}{$\beta$-Alanine $(\mathrm{mmol} / \mathrm{kg})$} \\
\cline { 2 - 6 } & \multicolumn{1}{c}{0} & 0.176 & 0.88 & \multicolumn{1}{c}{4.4} & 22 \\
\hline Initial Body weight $(\mathrm{g})$ & $52 \pm 1$ & $52 \pm 1$ & $51 \pm 2$ & $52 \pm 1$ & $53 \pm 1$ \\
Feed consumption (g/5 days) & $109 \pm 8$ & $111 \pm 4$ & $105 \pm 4$ & $110 \pm 3$ & $107 \pm 4$ \\
Body weight gain (g/5 days) & $108 \pm 7$ & $111 \pm 4$ & $110 \pm 5$ & $111 \pm 3$ & $105 \pm 2$ \\
Feed efficiency & $1.01 \pm 0.02$ & $1.00 \pm 0.02$ & $1.00 \pm 0.03$ & $1.00 \pm 0.01$ & $1.02 \pm 0.03$ \\
(Body weight gain/feed consumption) & $3.69 \pm 0.37$ & $3.86 \pm 0.31$ & $3.11 \pm 0.21$ & $3.62 \pm 0.25$ & $3.21 \pm 0.29$ \\
MPS weight (g) & $1.29 \pm 0.02$ & $1.25 \pm 0.02$ & $1.25 \pm 0.04$ & $1.26 \pm 0.04$ & $1.27 \pm 0.02$ \\
Whole brain weight (g) & & &
\end{tabular}

$\beta$-Alanine was orally administered twice a day for five days. MPS indicates Musculus pectoralis superficialis. For MPS weight, the right side was weighed and extrapolated to the whole weight. Values are means \pm standard error of the mean.

Table 2. Effect of $\beta$-alanine on amino acid and dipeptide levels in broiler chicks

\begin{tabular}{|c|c|c|c|c|c|c|}
\hline \multirow{2}{*}{ Tissue } & \multirow{2}{*}{ Contents } & \multicolumn{5}{|c|}{$\beta$-Alanine $(\mathrm{mmol} / \mathrm{kg})$} \\
\hline & & 0 & 0.176 & 0.88 & 4.4 & 22 \\
\hline \multirow[t]{6}{*}{ Brain } & Carnosine & $27 \pm 5$ & $27 \pm 7$ & $39 \pm 5$ & $35 \pm 4$ & $49 \pm 12$ \\
\hline & Anserine & $298 \pm 21$ & $304 \pm 32$ & $328 \pm 28$ & $378 \pm 28$ & $370 \pm 45$ \\
\hline & $\beta$-Alanine & $28 \pm 2$ & $30 \pm 2$ & $29 \pm 2$ & $32 \pm 2$ & $51 \pm 8$ \\
\hline & Histidine & $70 \pm 4$ & $65 \pm 7$ & $74 \pm 5$ & $82 \pm 6$ & $79 \pm 10$ \\
\hline & 1-Methylhistidine & $27 \pm 5$ & $22 \pm 5$ & $20 \pm 5$ & $25 \pm 4$ & $18 \pm 3$ \\
\hline & Taurine & $5180 \pm 294$ & $5111 \pm 358$ & $5092 \pm 288$ & $4664 \pm 338$ & $3929 \pm 261$ \\
\hline \multirow[t]{6}{*}{ MPS } & Carnosine & $6877 \pm 856$ & $6379 \pm 1258$ & $6116 \pm 878$ & $9162 \pm 1453$ & $11396 \pm 501$ \\
\hline & Anserine & $7380 \pm 585$ & $7699 \pm 644$ & $6882 \pm 532$ & $7611 \pm 635$ & $7236 \pm 699$ \\
\hline & $\beta$-Alanine & $83 \pm 10$ & $113 \pm 44$ & $109 \pm 17$ & $109 \pm 16$ & $264 \pm 54$ \\
\hline & Histidine & $258 \pm 19$ & $244 \pm 17$ & $256 \pm 17$ & $312 \pm 27$ & $292 \pm 29$ \\
\hline & 1-Methylhistidine & $105 \pm 16$ & $100 \pm 15$ & $86 \pm 14$ & $100 \pm 17$ & $69 \pm 11$ \\
\hline & Taurine & $2721 \pm 121$ & $2787 \pm 199$ & $2655 \pm 164$ & $2484 \pm 224$ & $1627 \pm 101$ \\
\hline \multirow[t]{6}{*}{ Plasma } & Carnosine & $7 \pm 1$ & $6 \pm 1$ & $7 \pm 1$ & $11 \pm 3$ & $13 \pm 1$ \\
\hline & Anserine & $14 \pm 2$ & $12 \pm 1$ & $15 \pm 5$ & $16 \pm 4$ & $18 \pm 2$ \\
\hline & $\beta$-Alanine & $11 \pm 2$ & $16 \pm 5$ & $11 \pm 1$ & $16 \pm 3$ & $56 \pm 5$ \\
\hline & Histidine & $98 \pm 13$ & $87 \pm 6$ & $102 \pm 10$ & $112 \pm 8$ & $122 \pm 21$ \\
\hline & 1-Methylhistidine & $41 \pm 6$ & $33 \pm 4$ & $25 \pm 3$ & $32 \pm 4$ & $30 \pm 7$ \\
\hline & Taurine & $354 \pm 29$ & $332 \pm 29$ & $325 \pm 26$ & $269 \pm 25$ & $199 \pm 19$ \\
\hline
\end{tabular}

$\beta$-Alanine was orally administered twice a day for five days. MPS indicates Musculus pectoralis superficialis. Values are means \pm standard error of the mean. Brain and MPS levels are in $\mathrm{nmol} / \mathrm{g}$ wet tissue. Plasma level is in $\mu \mathrm{M}$.

Table 3. Regression analysis of the effect of $\beta$-alanine on tissue carnosine, $\beta$-alanine or taurine level in broiler chicks

\begin{tabular}{|c|c|c|c|c|}
\hline Tissue & Contents & Regression equation & $\mathrm{R}^{2}$ & $P$ value \\
\hline \multirow[t]{3}{*}{ Brain } & Carnosine & $\mathrm{Y}=31.7(\mathrm{SE} 3.5)+0.8(\mathrm{SE} 0.4) \mathrm{X}$ & 0.16 & $<0.05$ \\
\hline & $\beta$-Alanine & $\mathrm{Y}=28.7(\mathrm{SE} 1.9)+1(\mathrm{SE} 0.2) \mathrm{X}$ & 0.457 & $<0.0001$ \\
\hline & Taurine & $Y=5092(\mathrm{SE} 160)-55(\mathrm{SE} 17) \mathrm{X}$ & 0.258 & $<0.005$ \\
\hline \multirow[t]{3}{*}{ MPS } & Carnosine & $\mathrm{Y}=6763(\mathrm{SE} 572)+224(\mathrm{SE} 59) \mathrm{X}$ & 0.313 & $<0.001$ \\
\hline & $\beta$-Alanine & $\mathrm{Y}=94.4(\mathrm{SE} 16.5)+7.5(\mathrm{SE} 1.7) \mathrm{X}$ & 0.383 & $<0.0001$ \\
\hline & Taurine & $\mathrm{Y}=2732(\mathrm{SE} 88)-51(\mathrm{SE} 9) \mathrm{X}$ & 0.494 & $<0.0001$ \\
\hline \multirow[t]{3}{*}{ Plasma } & Carnosine & $\mathrm{Y}=7(\mathrm{SE} 0.9)+0.3(\mathrm{SE} 0.1) \mathrm{X}$ & 0.229 & $<0.005$ \\
\hline & $\beta$-Alanine & $\mathrm{Y}=11.1(\mathrm{SE} 1.8)+2(\mathrm{SE} 0.2) \mathrm{X}$ & 0.781 & $<0.0001$ \\
\hline & Taurine & $\mathrm{Y}=329(\mathrm{SE} 14)-6(\mathrm{SE} 1) \mathrm{X}$ & 0.381 & $<0.001$ \\
\hline
\end{tabular}

$\beta$-Alanine was orally administered twice a day for five days. $\mathrm{Y}$ indicates level in each tissue $(\mu \mathrm{mol} / \mathrm{g}$ wet tissue) or in plasma $(\mu \mathrm{M})$. X indicates dose of administered $\beta$-alanine. MPS indicates Musculus pectoralis superficialis. 
the layer-type chicks). Regulation of carnosine and anserine metabolisms may be somehow different between layer-type and broiler-type chicks. Indeed, brain and MPS levels of these dipeptides and their constituents in the control group differed from those in the control group of layer-type chicks of the same age in the previous study, for example, $\beta$-Ala level of the MPS in layer-type chicks is approximately 2fold higher than that in broiler-type chicks (Tomonaga et al., 2005b). Clarification of the difference in these dipeptide metabolisms between broiler- and layer-type chicks may be useful for breeding because it may help to select chickens that contain dipeptides in high levels. On the other hand, the effectiveness in increasing dipeptide levels may be higher in younger chicks because we previously confirmed that dietary $\beta$-Ala $(0.5,1$ and $2 \%$ in commercial diet, from 4 weeks old for 4 weeks) enhances brain, but not muscle (MPS, Musculus pectoralis profundus, and Musculus biceps femoris), dipeptide levels in broilers (Tomonaga et al., 2006). To improve present method, it can be speculated that subsequent cessation for several days may be effective in increasing both dipeptide levels because intraperitoneal injections of $\beta$-Ala increased carnosine concentrations in the olfactory bulb in the brain and in gastrocnemius muscle and subsequent cessation of $\beta$-Ala treatment for 5 days caused an increase of anserine concentration in the gastrocnemius muscle in mice (Margolis et al., 1985). On the other hand, as shown in studies in horses (Dunnett and Harris, 1999), supplementation with $\beta$-Ala plus His may be more effective than supplementation with $\beta$-Ala alone. Further studies are necessary.

$\beta$-Ala levels of brain, MPS and plasma were dose-dependently increased while their taurine levels were decreased in a dose-dependent manner. Taurine depletion was commonly observed following $\beta$-Ala treatment because $\beta$-Ala competes with taurine for uptake (Hu et al., 2000; Tomonaga et al., 2005b, 2006). The supplemental $\beta$-Ala in the present study successfully competed with taurine. It is not clear why not only tissue but also plasma taurine level decreased. We hypothesized that temporarily increased taurine in the plasma might accelerate some physiological mechanisms of taurine excretion and as a result, plasma taurine decreased too much. Further study should be done to clarify this hypothesis. As pointed out in the introduction, tissue taurine depletion could induce physiological dysfunctions such as growth retardation (Sturman, 1993; Hu et al., 2000). In the present study, no significant negative effects were observed in growth parameters such as feed consumption, body weight gain, feed efficiency, MPS weight and whole brain weight (Table 1). On the other hand, as shown in the results section, two chicks died in higher $\beta$-Ala groups. Therefore, further study should be done to clarify whether present method influences the health in chickens.

Thus, supplemental $\beta$-Ala given to broiler chicks seems effective in increasing the carnosine in their MPS and brain. This may improve brain function and/or increase the commercial value of meat. To effectively increase the dipeptides in chickens, further nutritional studies are needed. In addition, other methods, such as stimulation of carnosine syn- thesis and/or suppression of its degradation by a method of molecular biology, may be effective. In particular, carnosine synthase was recently identified in chickens (Drozak et al., 2010) and this may make molecular studies easier to perform. Further studies are needed to determine how to increase dipeptides in broilers.

\section{Acknowledgments}

The costs of publication were supported in part by the Research Grant for Young Investigators of Faculty of Agriculture, Kyushu University. This work was supported by a Grant-in-Aid for Scientific Research (No. 22780740) from the Japan Society for the Promotion of Science and the Kieikai Research Foundation.

\section{References}

Aristoy MC and Toldra F. Histidine dipeptides HPLC-based test for the detection of mammalian origin proteins in feeds for ruminants. Meat Science, 67: 211-217. 2004.

Boldyrev AA and Severin SE. The histidine-containing dipeptides, carnosine and anserine: distribution, properties, and biological significance. Advances in Enzyme Regulation, 30: 175-194. 1990.

Derave W, Ozdemir MS, Harris RC, Pottier A, Reyngoudt H, Koppo $\mathrm{K}$, Wise JA and Achten E. $\beta$-Alanine supplementation augments muscle carnosine content and attenuates fatigue during repeated isokinetic contraction bouts in trained sprinters. Journal of Applied Physiology, 103: 1736-1743. 2007.

Djenane D, Martínez L, Sánchez-Escalante A, Beltrán JA and Roncalés P. Antioxidant effect of carnosine and carnitine in fresh beef steaks stored under modified atmosphere. Food Chemistry, 85: 453-459. 2004.

Dunnett $\mathrm{M}$ and Harris RC. Influence of oral $\beta$-alanine and Lhistidine supplementation on the carnosine content of the gluteus medius. Equine Veterinary Journal. Supplement, 30: 499-504. 1999.

Drozak J, Veiga-da-Cunha M, Vertommen D, Stroobant V and Van Schaftingen E. Molecular identification of carnosine synthase as ATP-grasp domain-containing protein 1 (ATPGD1). Journal of Biological Chemistry, 285: 9346-9356. 2010.

Gallant S, Semyonova M and Yuneva M. Carnosine as a potential anti-senescence drug. Biochemistry (Moscow), 65: 866-868. 2000.

Horinishi H, Grillo M and Margolis FL. Purification and characterization of carnosine synthetase from mouse olfactory bulbs. Journal of Neurochemistry, 31: 909-919. 1978.

Hu JM, Rho JY, Suzuki M, Nishihara M and Takahashi M. Effect of taurine in rat milk on the growth of offspring. Journal of Veterinary Medical Science, 62: 693-698. 2000.

Kohen R, Yamamoto Y, Cundy $\mathrm{KC}$ and Ames BN. Antioxidant activity of carnosine, homocarnosine, and anserine present in muscle and brain. Proceedings of the National Academy of Sciences of the United States of America, 85: 3175-3179. 1988.

Margolis FL, Grillo M, Kawano T and Farbman AI. Carnosine synthesis in olfactory tissue during ontogeny: effect of exogenous $\beta$-alanine. Journal of Neurochemistry, 44: 14591464. 1985.

Niijima A, Okui T, Matsumura Y, Yamano T, Tsuruoka N, Kiso Y and Nagai K. Effects of L-carnosine on renal sympathetic nerve 
activity and DOCA-salt hypertension in rats. Autonomic Neuroscience, 97: 99-102. 2002.

Nishimura T, Rhue MR, Okitani A and Kato H. Components contributing to the improvement of meat taste during storage. Agricultural and Biological Chemistry, 52: 2323-2330. 1988.

Pegova A, Abe $\mathrm{H}$ and Boldyrev A. Hydrolysis of carnosine and related compounds by mammalian carnosinases. Comparative Biochemistry and Physiology. B, Comparative biochemistry, 127: 443-446. 2000.

Statistical Analysis Systems (SAS). Stat View, Version 5. SAS Institute, Cary, NC. 1998.

Sturman JA. Taurine in development. Physiological Reviews, 73: 119-147. 1993.

Tomonaga S, Tachibana T, Takagi T, Saito ES, Zhang R, Denbow DM and Furuse M. Effect of central administration of carnosine and its constituents on behaviors in chicks. Brain Research Bulletin, 63: 75-82. 2004.

Tomonaga S, Tachibana T, Takahashi H, Sato M, Denbow DM and Furuse M. Nitric oxide involves in carnosine-induced hyper- activity in chicks. European Journal of Pharmacology, 524: 84-88. 2005a.

Tomonaga S, Kaji Y, Tachibana T, Denbow DM and Furuse M. Oral administration of $\beta$-alanine modifies carnosine concentrations in the muscles and brains of chickens. Animal Science Journal, 76: 249-254. 2005b.

Tomonaga S, Kaneko K, Kaji Y, Kido Y, Denbow DM and Furuse M. Dietary $\beta$-alanine enhances brain, but not muscle, carnosine and anserine concentrations in broilers. Animal Science Journal, 76: 79-86. 2006.

Tomonaga S, Yamane H, Onitsuka E, Yamada S, Sato M, Takahata Y, Morimatsu F and Furuse M. Carnosine-induced antidepressant-like activity in rats. Pharmacology Biochemistry and Behavior, 89: 627-632. 2008.

Yamano T, Niijima A, Iimori S, Tsuruoka N, Kiso Y and Nagai K. Effect of L-carnosine on the hyperglycemia caused by intracranial injection of 2-deoxy-D-glucose in rats. Neuroscience Letters, 313: 78-82. 2001. 